\title{
Decision-Making Using Efficient Confidence-Intervals with Meta-Analysis of Spatial Panel Data for Socioeconomic Development Project-Managers
}

\author{
Ashok Sahai \\ Department of Mathematics and Statistics, The University of the West Indies, St. Augustine Campus @ TRINIDAD \\ Email:Ashok.sahai@sta.uwi.edu \\ Clement K. Sankat \\ PVC/PRINCIPAL, The University of the West Indies, St. Augustine Campus @ TRINIDAD \\ Email: clement.sankat@sta.uwi.edu

\begin{abstract}
Koffka Khan
Department of Computing and Information Technology, The University of the West Indies, St. Augustine Campus @ TRINIDAD

Email: koffka.khan@sta.uwi.edu
\end{abstract}

\begin{abstract}
It is quite common to have access to geospatial (temporal/spatial) panel data generated by a set of similar data for analyses in a meta-data setup. Within this context, researchers often employ pooling methods to evaluate the efficacy of meta-data analysis. One of the simplest techniques used to combine individual-study results is the fixed-effects model, which assumes that a true-effect is equal for all studies. An alternative, and intuitively-more-appealing method, is the random-effects model. A paper was presented by the first author, and his co-authors addressing the efficient estimation problem, using this method in the aforesaid meta-data setup of the 'Geospatial Data' at hand, in Map World Forum meeting in 2007 at Hyderabad; INDIA. The purpose of this paper had been to address the estimation problem of the fixed-effects model and to present a simulation study of an efficient confidence-interval estimation of a mean true-effect using the panel-data and a random-effects model, too in order to establish appropriate 'confidence interval' estimation for being readily usable in a decisionmakers' setup. The present paper continues the same perspective, and proposes a much more efficient estimation strategy furthering the gainful use of the 'Geospatial Panel-Data' in the Global/Continental/ Regiona1/National contexts of "Socioeconomic \& other Developmental Issues'. The 'Statistical Efficient Confidence Interval Estimation Theme' of the paper(s) has a wider a mbit than its applicability in the context of 'Socioeconomic Development' only. This 'Statistical Theme' is, as such, equally gainfully applicable to any area of application in the present world-order at large inasmuch as the "Data-Mapping" in any context, for
\end{abstract}

example, the issues in the topically significant area of "Global Environmental Pollution-Mitigation for Arresting the Critical phenomenon of Global Warming". Such similar issues are tackle-able more readily, as the impactful advances in the "GIS \& GPS" technologies have led to the concept of "Managing Global Village" in terms of 'Geospatial Meta-Data'. This last fact has been seminal to special zeal-n-motivation to the authors to have worked for this improved paper containing rather a much more efficient strategy of confidenceinterval estimation for decision-making team of managers for any impugned area of application.

Index Terms-Geospatial, Meta-Data, Confidence Interval, Socioeconomic, Data Mapping

\section{Introduction}

Meta-analysis represents the statistical analysis of a collection of analytic results motivated by the gainful urge to integrate the findings in the context of geospatial data that represent environmental and socioeconomic indicators in a given context. This paper is in sequel to [8]. It is quite common to have access to panel data generated by a set of similar spatial data for analyses in a meta-data setup. Within this context, researchers often employ pooling methods to evaluate the efficacy of metadata analysis. One of the simplest techniques used to combine individual study results is the fixed-effects model, which assumes that a true effect is equal for all studies. An alternative, and intuitively more appealing method, is the random-effects model. 
The purpose of this paper is to address the estimation problem of the fixed effects model and to present a simulation study of an efficient estimation of a mean true effect using panel data and a random effects model in order to establish appropriate 'Confidence Interval'(CI) estimation for being readily usable in a decision-makers' setup..

In that paper of [8], is attempted to be improved by proposing a method of statistical estimation aimed at improving the accuracy of the resultant CIs for an efficient estimation of a mean true effect using panel data and a random effects model. The improvement of the reliability, which has been demonstrated by the use of a simulation study, is two-fold namely mathematical statistically using the concept of the 'Minimum Mean Square Estimator (MMSE)' of the reciprocal of the heterogeneities [using the 'Bootstrapping'] of the studies, and by computing the simulational averages and hence the "Relative Efficiency (REff.)' of the estimator(s), using '11,000' replications in the simulation. Bootstrapping is the well-known technique of 'Data Mining' [Please peruse the relevant details in 'APPENDIX \# 2' in this paper].

To illustrate the universal applicability of the impugned technique of 'Meta-Analys is of the PanelData' targeted in this paper in the areas additional to that of the socio-economic studies, as well-illustrated in the [8]'s paper, we take the example of the currently important 'Global Warming Problem \& Environmental Pollution'. For the 'Decision-Making Managers' at United Nation, we could construct the handy CIs (Confidence-Intervals) for their decision-making and the judgment regarding the allocations of the combating-resources to fight-down the awe-fully significant environ mental-pollution-threat to humanity, including the moneys to the various countries/ continents on the earth, we would do well to make good use of the 'Geospatial Panel Data'. The Meta-Analysis would have to be carried up in a hierarchical-setup. The Overall Global CIs would also be available finally to the decision-manager @ UN consequent upon this hierarchally carried out meta-analysis. The Final Global Meta-Analysis could have been using the 'Continental Geospatial Data'; The Continental Meta-Analysis could have, well, been using the data for various countries in the continent; The Country-wise Meta-Analysis could well be using the Meta-Data of various states in the country, and finally the state-wise Meta-Analys is would have to be using the Geospatial Meta-Data of the various cities and the counties therein! At the grass-root level as well, we might well use the 'Geo-Temporal Data'. For example, for the study of the 'Environmental Pollution' in a city by the harmful emissions from the automobiles commuting therein, the Data would vary temporally at the peak/ moderate/ less-busy hours of the day/ night, etc., etc! It would be in place to mention that the cases of missing/ poor data in underdeveloped/ developing countries in the aforesaid context could well be dealt-with by generating the relevant synthetic data to take care of such Geo-Spatial-Temporal Data Gaps through the use of well-known powerful statistical techniques.

Incidentally, such meta-analyses of 'panel-data' are gaining quick-currency amongst medical researchers, and are becoming increasingly popular day-by-day in their research investigations, where information on efficacy of a treatment is available from a number of clinical studies with similar treatment protocols. Often, when one study is considered separately, the data therein (as generated per the randomized control clinical trial(s)) would be rather too small or too limited in its scope to lead to unequivocal or generalizable conclusions concerning, for example, the effect of the socio-economic co-variates under investigation, in the target study of the predecessor-paper.

A number of methods are available to set up the confidence limits for the overall mean effect(s) for the meta-analysis of the panel-data in the context of a random/fixed effects model generated by these data on the socio-economic variable(s). A popular and simple commonly-used method is the one proposed by the [4] approach. It is worth noting, in the context of panel data/ meta-analysis, that the simplest statistical technique for combining the individual study results is based on a fixed effects model. In the fixed effects model, it is assumed that the true effect is the same for all studies generating the panel data. On the other hand, a random effects model allows for the variation in the true-effect across these studies in various states (in a federal setup like in U.S.A./India, e.g.)/ districts/ Counties/municipalities/village panchayat/block therein, and is, therefore, more realistic a model.[5], in a systematic search of the first ten issues published in 1982 of each of the four weekly journals (NEJM, JAMA, BMJ, and Lancet) found only one article (out of 589) that considered combining results using formal statistical methods. The basic difficulty one faces in trying to combine/integrate the results from various studies is generated by the diversity amongst these studies at hand in terms of the methods employed therein as also the design of these studies Also due to different parent populations and varying sample sizes, each study has a different level of sampling error. Hence, while integrating the results from such varied studies, one ought to assign varying weights to the information stemming from respective studies; these weights reflecting the relative 'value' of each of this information. In this context, [1] highlighted the need for the careful considerations in developing the methods for drawing inferences from such heterogeneous, though logically related, studies. [2] Observed that, in this setting, it would be more appropriate to use a regression analysis to characterize the differences in study outcomes.

In the context of a random effects model for the meta/panel-data analys is, there are a number of methods available to construct the confidence limits for the overall mean effects. [9] Proposed a simple confidence 
interval for meta-analysis, based on the t-distribution. Their approach, as per the simulation study, is quite likely to improve the coverage probability of the [4]'s approach. In the present paper we propose a couple of more efficient constructions of this confidence interval. A simulation study is carried out to demonstrate that our propositions improve the coverage probability of both of the aforesaid methods.

Meta-analysis represents the statistical analysis of a collection of analytic results motivated by the gainful urge to integrate the findings in the context of geospatial data that represents socio-economic indicators in a given context. We address the problem in two steps. First, we explore the current state of geo-spatial representation and its acquisition cost, which is often a barrier to implementing such studies. In this part, we explore the different methods of mapping and the possible alternatives for doing so. In the next part, we address the statistical approach of meta-analys is and the appropriate use of confidence intervals. Our goal in this paper is to propose a platform for mapping geo-spatial data generated by multiple socio-economic studies in India using freely available services and/or open source software. The data usually available for such representations is not suitable for direct analysis and representation. We aim to use meta-analysis techniques to improve the suitability of data for geo-spatial applications.

\subsection{Geo-spatial Mapping}

Mapping of spatial data has been investigated in many contexts. Lately though, access to mapping technology and geo-spatial software has given rise to applications called mashups (). In a mashup application, the mapping engine is provided by an independent service provider, and the spatial data typically comes from an independent source. The geo-spatial data is layered over an existing mapping structure to provide a composite map.

One example of such an application in the US context is Housing Maps (). This mashup application utilizes Google, Inc.'s mapping technology by accessing its published Application Programming Interfaces (API). Via this API, Housing Maps passes street-level address coordinates and related information (housing prices in this case) to the Google service, thereby creating a housing map. The data for housing comes from yet another freely-accessible service called Craigslist. This service allows interested landlords to post their real estate properties for rent or sale. The owner posts a location, price and description. Craigslist service then makes this information available via their website. As is evident from the following figure, the housing data is "mashed up" with the mapping service to create a composite application for geo-spatial representation of real estate in a given geographical locality (in this case, San Francisco, CA).

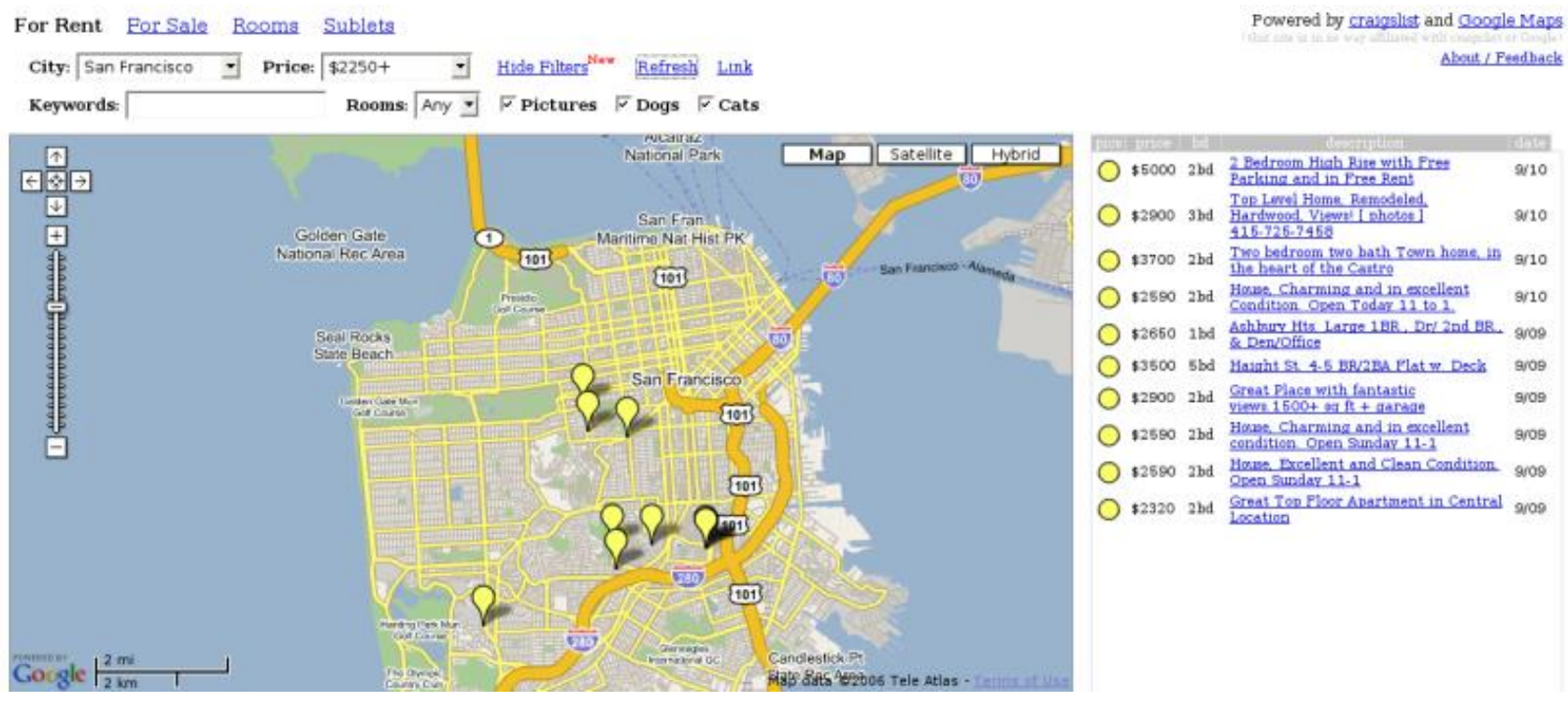

Fig. 1: JPDA algorithm flow chart

Data collected for many socio-economic or healthcare projects are often incomplete or inconsistent due to several issues such as data collection errors, social taboo or illiteracy reasons. Data collected from multiple sources can compound the problem, especially when we layer different data sources over a common geo-spatial space. Better estimators are needed for assessing the representation of data particu larly in cases where the point estimation holds little meaning and an interval provides more latitude for interpretation.
In the next section, we will look at a particular project and its data collection efforts.

\subsection{B.E-immunization}

This project is a relatively new approach for immunizing children against fatal childhood diseases in Khammam District in Andhra Pradesh. It has helped in bringing immunizations closer to the communities scattered in the hard-to-reach areas. The project's aim is 
to increase the reach of immunization in developing countries with the help of Information and Communication Technology. Health workers capture details on a handheld device at the point of immunization. After giving the shot to a baby, the health worker updates the shot history. A receipt is also generated for the parents.

The project was initiated by Rajendra Nimje, a research fellow at Stanford University. From a news item on the project: Khammam Collector Rajendra Narendra Nimje, who was a "digital vision fellow" of Stanford University during 2003-2004, had undertaken the project as part of his fellowship. The prototype of the concept was developed and tested in Nalgonda district from December 2003 to April 2004. Egged on by the success, the e-immunization was taken up in select villages. People of Edu llacehruvu, Ramana Tanda, Bisarajapalli Tanda, Balajinagar Tanda, Balam Tanda under the Tirumalayapalem sub centre and Erragadda, Kokkireni, Tallacheruvu, Gopalapuram, Timmakkapeta villages under Kokkireni sub centers were the first in the district to enjoy the benefits of the e-immunization programme launched about a year ago (on March 9, 2005).

This project is a prime example of data being generated on the field by trained and untrained individuals. In this case, a certain degree of automation will help in reducing errors during data collection. Data from this project can be combined with census data from the same district and be mapped on a geo-spatial platform, thereby providing us with an immunization density map of the district of Khammam. The mapping of such data will provide with interesting insights, but point estimates are not very meaningful in this context. A better estimator of such efforts would be an interval estimate. We address this problem in the next sections.

\section{The Problem Formulation}

The statistical inference problem is concerned with using the information from ' $\mathrm{k}$ ' independent studies in the meta-analyses setup. Set the random variable ' $y_{i}$ ' to stand for the effect size estimate from the 'ith.' study. It would be in order to note here that, some commonly used, measures of effect size are mean difference, standardized mean difference, risk difference, relative risk, and odds-ratio. As the 'Odds-Ratio (OR)', which is of particu lar use in retrospective or case-control studies, is mostly used, we would confine to it for the simplicity of illustration in our paper. Nevertheless, it is without any loss of generality inasmuch as the details of this paper are analogously valid for the other measures of effect size.

Let $\mathrm{n}_{\mathrm{ti}}$ and $\mathrm{n}_{\mathrm{ci}}$ denote the sample sizes and let $\mathrm{p}_{\mathrm{ti}}$ and $\mathrm{p}_{\mathrm{ci}}$ denote the proportions dying (not achieving the stipulated goal) for each of the treatment (t) and control (c) groups, where 'i' stands for the designation of the study number: 'I $=1$ (1) n'. Also, let $\mathrm{x}_{\mathrm{ti}}$ and $\mathrm{x}_{\mathrm{ci}}$ denote the observed number of the deaths for the treatment and the control groups respectively, for the study number ' $i$ '. We note that for the 'ith.' study the following gives the observed log-odds ratio $\left(\log \left(\mathrm{OR}_{\mathrm{i}}\right)\right)$ and the corresponding estimated variance.

$$
\mathrm{OR}_{\mathrm{i}}=\frac{\left(1-\mathrm{p}_{\mathrm{ci}}\right) \cdot \mathrm{p}_{\mathrm{ti}}}{\mathrm{p}_{\mathrm{ci}} \cdot\left(1-\mathrm{p}_{\mathrm{ti}}\right)}
$$

$\operatorname{estimated}\left(\sigma_{\mathrm{i}}\right)^{2} \equiv\left(\sigma_{\mathrm{i}}\right)^{2}==$
estimated $\left(\operatorname{var}\left(\log \left(\mathrm{OR}_{\mathrm{i}}\right)\right)\right)=\frac{1}{\mathrm{x}_{\mathrm{ti}}}+\frac{1}{\left(\mathrm{n}_{\mathrm{ti}}-\mathrm{x}_{\mathrm{ti}}\right)}+\frac{1}{\mathrm{x}_{\mathrm{ci}}}+\frac{1}{\left(\mathrm{n}_{\mathrm{ci}}-\mathrm{x}_{\mathrm{ci}}\right)}$

The important point to be noted at this stage is that the estimated $\left(\sigma_{i}\right)^{2}$ is rather a very close estimate of the respective population variance $\left(\sigma_{\mathrm{i}}\right)^{2}$, and that it is analogously closely available for the population variances for the cases of other measures of the effect size. For example, if the effect size $y_{i}$ happens to be the difference in proportions, ' $\mathrm{p}_{\mathrm{ti}}-\mathrm{p}_{\mathrm{ci}}$ ', we estimate the population variance $\left(\sigma_{i}\right)^{2}$ by:

$$
\operatorname{Estimated}\left(\sigma_{i}\right)^{2} \equiv\left(\sigma_{i}\right)^{2}=p_{t i}\left(1-p_{t i}\right) / p_{C i}\left(1-p_{C i}\right)
$$

Now, we might note that the general model is specified as follows.

Wherein,

$$
y_{i}=\theta_{i}+\varepsilon_{i}, i=1, \ldots, k
$$

And,

$$
\varepsilon_{\mathrm{i}} \approx \mathrm{N}\left(0, \sigma_{\mathrm{i}}^{2}\right)
$$

Wherein

$$
\theta_{\mathrm{i}}=\mu+\partial_{\mathrm{i}}
$$

$$
\partial_{\mathrm{i}} \approx \mathrm{N}\left(0, \tau^{2}\right)
$$

Hence, essentially the model comes to be:

$$
\mathrm{y}_{\mathrm{i}}=\mu+\partial_{\mathrm{i}}+\varepsilon_{\mathrm{i}} ; \mathrm{i}=1, \ldots, \mathrm{k}
$$

It is also important to note that whereas $\partial_{i}$ stands for the random error across the studies, $\varepsilon_{\mathrm{i}}$ represents the random error within a study, and that $\partial_{\mathrm{i}}$ and $\varepsilon_{\mathrm{i}}$ are assumed to be independent. Also, the parameter $\tau^{2}$ is a measure of the heterogeneity between the ' $\mathrm{k}$ ' studies. We will refer to it in our paper as the 'heterogeneity variance', which it is often called by.

Perhaps the important and the most crucial element in the panel-data/meta analysis is the challenge of developing an efficient estimator of this heterogeneity variance' $\tau^{2}$. [4] Proposed and used the following estimate:

Estimate of $\left(\tau^{2}\right)=$

$$
\max \left\{0, \frac{\sum_{i=1}^{\mathrm{k}} \mathrm{w}_{\mathrm{i}}\left(\mathrm{y}_{\mathrm{i}}-\hat{\mu}\right)^{2}-(\mathrm{k}-1)}{\sum_{\mathrm{i}=1}^{\mathrm{k}} \mathrm{w}_{\mathrm{i}}-\sum_{\mathrm{i}=1}^{\mathrm{k}} \mathrm{w}_{\mathrm{i}}^{2} / \sum_{\mathrm{i}=1}^{\mathrm{k}} \mathrm{w}_{\mathrm{i}}}\right\}
$$


Wherein, $w_{i}=1 / \sigma_{i}^{2}$ and the weighted estimate of the mean effect is given by:

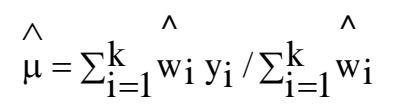

Also, herein the weight ' $\mathrm{w}_{\mathrm{i}}$ ' is assumed to be known.

Earlier, we noted that the estimated $\left(\sigma_{\mathrm{i}}\right)^{2}$ is rather a very close estimate of the respective population variance $\left(\sigma_{\mathrm{i}}\right)^{2}$. Therefore, most usually the sample estimate $\left.\hat{\sigma}_{\mathrm{i}}\right)^{2}$ is used in place of $\left(\sigma_{\mathrm{i}}\right)^{2}$, so that $\mathrm{w}_{\mathrm{i}}=1 /\left(\sigma_{\mathrm{i}}\right)^{2}$ is used in (1), and estimated $w_{i}$, i.e. $\hat{w}_{i}=1 /\left(\hat{\sigma}_{i}\right)^{2}$ in (2).

Recently, [9] proposed a simple confidence interval for the meta-analysis. This approach, consisting in the construction of the confidence interval based on the $t$ distribution', significantly improved the 'coverage probability' compared to the existing 'most popular' [4]'s approach, as outlined above.

It is worth noting, in the above context, that recently [3] presented a comprehensive summary of the existing methods of constructing the confidence interval for meta-analysis and carried out their comparisons in terms of their 'coverage probabilities'.

While, the most-commonly-used/popular method of [4] random effects method led to the coverage probabilities below nominal level, the profile likelihood interval of [6] led to the higher coverage probabilities.

However, the profile likelihood approach happens to be quite cumbersome computationally, and involves an iterative calculation as does the 'simple likelihood method' presented in [3]. On the other hand, [3]'s proposition of a simple approach for the construction of a ' $100(1-\alpha)$ ' percent confidence interval for the overall effect in the random effects model, suing the pivotal inference based on the $t$-distribution, uses no iterative computation like the popular method of [4].

Moreover, the [9]'s proposition has a better 'coverage probability' than that of [4]. Consequently, while [4]'s confidence interval for meta-analysis used to be the most popular/commonly-used confidence interval, that of [9]'s happens to be rather-the-best one in terms of the most important count, namely that of the 'coverage probability', on which the 'confidence intervals' are compared and rated.

In fact, therefore, our motivation is basically to attempt the improvement of these two methods for constructing the 'Confidence Intervals' for an interval estimate for the overall mean effect across the ' $k$ ' studies, using the panel/meta-data generated by these studies. The impugned improvement was targeted mainly at the improved 'coverage probabilities'. It is amply achieved, as is revealed by the comparison using a 'Simulation Study'. The modified "[9] Estimator (MSJE)" proposed in this paper turns up to be the best to use.

\section{The Proposed Confidence Interval Estimates}

As noted in the last section, the important and the most crucial element in the panel-data/meta analysis is the challenge of developing an efficient estimator of this heterogeneity variance' $\tau^{2}$, [4]'s approximate $100(1-\alpha)$ per cent confidence interval for the general mean effect ' $\mu$ ', using the random effects model, is given by:

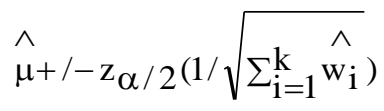



Also, $\hat{\mathrm{w}}_{\mathrm{i}}=1 /\left(\left(\hat{\sigma}_{\mathrm{i}}\right)^{2}+(\hat{\tau})^{2}\right)$ is evaluated using:

$$
\hat{(\tau)}^{2}=\max \left\{0, \frac{\sum_{\mathrm{i}=1}^{\mathrm{k}} \mathrm{w}_{\mathrm{i}}\left(\mathrm{y}_{\mathrm{i}}-\hat{\mu}\right)^{2}-(\mathrm{k}-1)}{\sum_{\mathrm{i}=1}^{\mathrm{k}} \mathrm{w}_{\mathrm{i}}-\sum_{\mathrm{i}=1}^{\mathrm{k}} \mathrm{w}_{\mathrm{i}}^{2} / \sum_{\mathrm{i}=1}^{\mathrm{k}} \mathrm{w}_{\mathrm{i}}}\right\}, \text { as in }(1) \text {. }
$$

It would be in order here to note that $z \alpha / 2$ in (3) above is the $\alpha / 2$ upper quantile of the standard normal variable. To construct an alternative simple confidence interval for the general mean effect ' $\mu$ ', using the random effects model, assuming that

$$
\mathrm{y}_{\mathrm{i}} \cong \mathrm{N}\left(\mu,\left(\sigma_{\mathrm{i}}\right)^{2}+(\tau)^{2}\right)
$$

Recently Sidik and Jonkman (2002) proposed an improvement. They, subject to the assumption that $\hat{w}_{i}=1 /\left(\sigma_{i}\right)^{2}$ 's correct weights ((i.e., essentially that $\hat{w}_{\mathrm{i}} \approx \mathrm{w}_{\mathrm{i}} \forall \mathrm{i}=1,2, . . \mathrm{k}$.), being close estimates), noted that:

$$
\mathrm{Z}_{\mathrm{W}}=(\hat{\mu}-\mu) /\left(1 / \sqrt{\left(\sum_{\mathrm{i}=1}^{\mathrm{k}} \hat{\mathrm{W}_{\mathrm{i}}}\right)}\right) \cong \mathrm{N}(0,1)
$$

and

$$
\mathrm{Q}_{\mathrm{W}}=\sum_{\mathrm{i}-1}^{\mathrm{k}} \hat{\mathrm{w}}_{\mathrm{i}}\left(\mathrm{y}_{\mathrm{i}}-\hat{\mu}\right)^{2} \cong \chi_{(\mathrm{k}-1)}^{2}
$$

They showed that $\mathrm{Zw}$ and Qw are independently distributed. Hence, it follows that:

$$
\frac{\hat{(\mu-\mu)} \sqrt{\left(\sum_{\mathrm{i}=1}^{\mathrm{k}} \hat{\mathrm{w}}_{\mathrm{i}}\right)}}{\sqrt{\left\{\sum_{\mathrm{i}=1}^{\left.\mathrm{k} \hat{w_{i}}\left(\mathrm{y}_{\mathrm{i}}-\hat{\mu}\right)^{2} /(\mathrm{k}-1)\right\}}\right.}} \cong \mathrm{t}_{(\mathrm{k}-1)}
$$


This, thence, led to [9]'s proposition of an approximate 100(1- $\alpha)$ per cent confidence interval for the general mean effect ' $\mu$ ', using the random effects model, is given by:



It would be in order here to note that $\mathrm{tk}-1, \alpha / 2$, in (6) above, is the $\alpha / 2$ upper quantile of the $t$-distribution with k-1 degrees of freedom. Also, under the assumption of known weights,

$$
\mathrm{Q}_{\mathrm{w}} /\left[(\mathbf{k}-\mathbf{1}) \sum_{\mathbf{i}=\mathbf{1}}^{\mathbf{k}} \hat{\mathbf{w}_{\mathbf{i}}}\right] \text { is an unbiased estimator of }
$$

the variance of $\hat{\mu}$

It is very significant fact at this stage to note that both [4]'s, as also [9]'s 100(1- $\alpha)$ per cent confidence interval for the general mean effect ' $\mu$ ', using the random effects model, are 'approximate', inasmuch as their validity is subject to the extent of the truth of the underlying assumption that the weights:

$$
\widehat{\mathrm{w}_{\mathrm{i}}} \approx \mathrm{w}_{\mathrm{i}} \forall \mathrm{i}=1,2, . . \mathrm{k} .
$$

and hence the $\left(\hat{\sigma_{i}}\right)^{2} \approx\left(\sigma_{i}\right)^{2}, \forall i=1,2, . . \mathrm{k}$.

Thus, essentially, it boils down to as to how efficient our estimate of the inter-study heterogeneity variance' $\tau^{2}$, is. We might as well note here that:

If the estimate of $\left(\tau^{2}\right)$, i.e. $(\hat{\tau})^{2}=0$, the rando m effects model boils down to the fixed-affect model.

Further, we might mention here that the more efficient estimation of the inter-study heterogeneity variance' $\tau^{2}$, is the key motivating factor for our propositions to possibly improve the 'coverage probability'. In both the papers, namely those of [4]and [9], the estimation of this inter-study heterogeneity variance' $\tau^{2}$, as is nicely described in [3], is as follows:

The impugned two-stage random effects model:

$$
\mathrm{y}_{\mathrm{i}}=\theta_{\mathrm{i}}+\varepsilon_{\mathrm{i}}, \mathrm{i}=1, \ldots, \mathrm{k}, \text { Wherein, } \varepsilon_{\mathrm{i}} \approx \mathrm{N}\left(0, \sigma_{\mathrm{i}}{ }^{2}\right)
$$

$\&$

$$
\theta_{\mathrm{i}}=\mu+\partial_{\mathrm{i}} \text {. Wherein } \partial_{\mathrm{i}} \approx \mathrm{N}\left(0, \tau^{2}\right) .
$$

That could well be re-written equivalently as:

$$
\begin{aligned}
& \quad y_{i}=\mu+\partial_{i}+\varepsilon_{i} ; i=1, \ldots, k, \text { Wherein } \varepsilon_{i} \approx N\left(0, \sigma_{i}^{2}\right) \\
& \text { and } \partial_{i} \approx N\left(0, \tau^{2}\right) .
\end{aligned}
$$

As noted earlier, under the assumptions that $\hat{w}_{i}=1 /\left(\sigma_{i}\right)^{2}$ 's correct weights ((i.e., essentially that $\hat{w}_{i} \approx w_{i} \forall i=1,2, . . k$. ), being close estimates) and that $\partial_{\mathrm{i}}$ and $\varepsilon_{\mathrm{i}}$ are independent (all assumptions being wellknown to be quite reasonably tenable), we have (to the extent of the approximation due to the extent of the tenability of the afores aid assumptions ):

$$
\hat{\mu}_{\tau}=\frac{\sum_{\mathrm{i}=1}^{\mathrm{k} \wedge} \mathrm{w}_{\mathrm{i}}(\tau) \mathrm{y}_{\mathrm{i}}}{\sum_{\mathrm{i}=1}^{\mathrm{k} \wedge} \mathrm{w}_{\mathrm{i}}(\tau)}
$$

With variance:

$$
\operatorname{var}\left(\hat{\mu}_{\tau}\right)=\frac{1}{\sum_{i=1}^{\mathrm{k} \hat{w_{i}}(\tau)}}
$$

In the above,

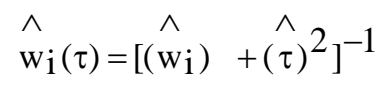

and

$$
\hat{\mathrm{w}}_{\mathrm{i}}=1 /\left(\sigma_{\mathrm{i}}\right)^{2} ; \mathrm{i}=1, \ldots, \mathrm{k}
$$

Now, assuming that $\tau^{2}$ is known, we have:

$$
\left.\hat{\mu}_{\tau} \cong \mathrm{N}(\mu, 1) / \sum_{\mathrm{i}=1}^{\mathrm{k}} \hat{\mathrm{w}}_{\mathrm{i}}(\tau)\right)
$$

It is interesting to note that the random effects model confidence intervals for $\mu$ are expected to be wider than those constructed under fixed effects model simply due to the facts that $\hat{\mu} \leq \hat{\mu}_{\tau}$, and hence

$$
\operatorname{var}(\hat{\mu}) \leq \operatorname{var}\left(\hat{\mu}_{\tau}\right)
$$

As $\tau^{2}$ is unknown in practice we ought to estimate it. [4] derived an estimate of $\tau^{2}$, using the meted of moments, by equating an estimate of the expected value of $\mathrm{Q}_{\mathrm{w}}$ with its observed value say, ' $\mathrm{q}_{\wedge}$ '.

$$
\mathrm{E}\left(\mathrm{Q}_{\mathrm{W}}\right)=\mathrm{k}-1+\tau^{2}\left(\sum_{\mathrm{l}=1}^{\mathrm{k}} \hat{\mathrm{wi}}-\frac{\sum_{\mathrm{l}=1}^{\mathrm{k}}\left(\mathrm{w}_{\mathrm{i}}\right)^{2}}{\sum_{\mathrm{l}=1}^{\mathrm{k}} \hat{\mathrm{wi}_{\mathrm{i}}}}\right)=\mathrm{q}_{\hat{\mathrm{w}}}
$$

Therefore, we note that if ' $t$ ' is the solution of the above equation, we have: 


$$
\mathrm{t}=\frac{\left[\mathrm{q}_{\wedge}-(\mathrm{k}-1)\right]}{\left[\sum_{\mathrm{l}=1}^{\mathrm{k}} \hat{\mathrm{w}_{\mathrm{i}}}-\frac{\left.\sum_{\mathrm{l}=1}^{\mathrm{k}} \hat{\left(\mathrm{w}_{\mathrm{i}}\right)^{2}}\right]}{\sum_{\mathrm{l}=1}^{\mathrm{k}} \hat{\mathrm{w}_{\mathrm{i}}}}\right.}
$$

So as to ward off the possibility of a negative value of 't' (which will be an unacceptable value of $\tau^{2}$, as any variance could not be negative), we define:

Estimated $\left(\tau^{2}\right)=\mathrm{t}$ if $\mathrm{t}>0$; and estimated $\left(\tau^{2}\right)=0$

if $\mathrm{t} \leq 0$

Using (11) in (9), we get the $\hat{\mathrm{w}}_{\mathrm{i}}(\tau)=\left[\left(\hat{\mathrm{w}}_{\mathrm{i}}\right)+(\hat{\tau})^{2}\right]^{-1}$ (where in $\left.\hat{w}_{i}=1 /\left(\hat{\sigma}_{i}\right)^{2}\right) ; i=1, \ldots, k$ to be used in (10), with the estimated variance of $\hat{\mu}_{\tau}$

$$
\begin{aligned}
& \hat{\operatorname{var}}\left(\hat{\mu}_{\tau}\right)=1 \\
& \underset{i=1}{\mathrm{k} \wedge} \hat{\mathrm{wi}}(\tau))
\end{aligned}
$$

Both, [4]'s and [9]'s propositions of an approximate $100(1-\alpha)$ per cent confidence interval for the general mean effect ' $\mu$ ', using the random effects model (as in (3) \& (6), respectively), use the $\hat{\mu}$ generated by the aforesaid and use the value of $\hat{\operatorname{var}\left(\hat{\mu}_{\tau}\right)}$, as in (13).

Essentially our proposition of the improved Confidence Interval (CIs) estimates of the general mean effect ' $\mu$ ' consist solely in a more efficient estimation of,$\hat{\operatorname{var}\left(\hat{\mu}_{\tau}\right)}$ ' in (13). For this purpose the following results are importantly relevant:

Lemma: If an estimate, say 's" (usual unbiased sample variance estimator) of the population variance, say ' $\sigma^{2}$ ' is based on a random sample $X_{1}, X_{2}, \ldots X_{k}$ from a Normal population $\mathrm{N}\left(\Theta, \sigma^{2}\right)$, we have: $\{(\mathrm{k}-$ 1). $\left.\mathrm{s}^{2}\right\} / \sigma^{2} \approx \chi_{(\mathrm{k}-1)}^{2}$ (: Chi-Square distribution on ' $(\mathrm{k}-1)$ ' degrees of freedom).

Further, we have: Minimum Mean Square Error Estimator MMSEE of ' $1 / \sigma^{2}$ is $1 /\left(\mathrm{k}^{*} \cdot \mathrm{s}^{2}\right)$.

$$
\text { Wherein } \mathrm{k}^{*}=(\mathrm{k}-1) /(\mathrm{k}-5)
$$

Proof: As, in the case of the random sample from a normal distribution, it is rather very well-known that the 'sample variance' $s^{2}$ is a 'complete sufficient statistic' for the 'population variance' $\sigma^{2}$. Therefore, Minimum Mean Square Error Estimator (MMSEE) of ' $1 / \sigma^{2}$, is simply its MMSE estimator of the class " $\mathrm{M} / \sigma^{2}$ ".

Now, we use the following equations, which are easily derived:

$$
\mathrm{E}\left[\left(1 / \mathrm{s}^{2}\right)\right]=[(\mathrm{k}-3) /(\mathrm{k}-1)] \cdot\left(1 / \sigma^{2}\right)
$$

$\&$

$$
\mathrm{E}\left[\left(1 / \mathrm{s}^{4}\right)\right]=\left[(\mathrm{k}-3) .(\mathrm{k}-5) /(\mathrm{k}-1)^{2}\right] \cdot\left(1 / \sigma^{4}\right)
$$

Hence, we could easily establish that the Minimum Mean Square Error Estimator (MMSEE) of ' $1 / \sigma^{2}$, would be as stated in (14). Q. E. D.

Hence, we propose the following modified more efficient Cis, modifying the say, "Original DerSimonian-Laird Estimator (ODLE) [4]”, and modifying the say. "Original Sidik-Jonkman Estimator (OSJE) [9]" defined, respectively, in (3) and (6) above.

We would call our estimators as the "Modified DerSimonian-Laird Estimator (MDLE) [4]", and as the "Modified Sidik-Jonkman Estimator (MSJE) [9]", respectively.

Essentially, the 'sole' difference between ODLE \& MDLE, as also between OSJE \& MSJE consists in replacing ' $\mathrm{k}$ ' in (3) and (6), respectively by ' $\mathrm{k}$ * for the modifications under the TWO approaches consisting in "UMVUE' estimation of " $1 / \sigma^{2}$, whereas ' $\sigma^{2}$ ' here in stands for the heterogeneity variance' $\tau^{2}$, , and the parameter $\tau^{2}$ is essentially a measure of the heterogeneity between the ' $k$ ' studies.

\section{The Simulation Study}

The format of the 'Simulation Study' in our paper to compare the "Original DerSimonian-Laird Estimator (ODLE) [4]" and the Original Sid ik-Jonkman Estimator (OSJE) [9]" with our estimators "Modified DerSimonian-Laird Estimator (MDLE) [4], and as the "Modified Sidik-Jonkman Estimator (MSJE) [9]", respectively, is the same as that in [9].

To compare the simple confidence interval based on the t-distribution with the DerSimonian and Laird interval in terms of coverage probability, we performed a simulation study of met analysis for the random effects model.

Throughout the study, the overall mean effect $\mu$ is fixed at 0.5 and the error probability of the confidence interval, $\alpha$, is set at 0.05 . We use only one value for $\mu$ because the t-distribution interval based on the pivotal quantity in (5) and the DerSimonian and Laird interval [4] are both invariant to a location shift. Three different values of $\tau^{2}$ are used: $0.05 ; 0.08$, and 0.1 . For each $\tau^{2}$, three different values of $\mathrm{k}$ (namely 10, 20, and 60 to keep the comparisons modestly) are considered. The number of simulation runs for the meta-analysis of $\mathrm{k}$ studies is 11000 . The simulation data for each run are generated in terms of the most popular measure of effect size in meta-analysis, the log of the odds ratio. That is, the generated effect size yi is interpreted as a log odds ratio (it could alternatively be the mean effect of the ith. Study, as well).

For given $k$, the within-study variance $\sigma_{i}^{2}$ is generated using the method of [3]. Specifically, a value 
is generated from a chi-square distribution with one degree of freedom, which is then scaled by $1=4$ and restricted to an interval between 0.009 and 0.6. This results in a bimodal distribution of $\sigma_{\mathrm{i}}{ }^{2}$, with the modes at each end of the distribution. As noted by [3], values generated in this way are consistent with a typical distribution of $\sigma_{i}^{2}$ for $\log$ odds ratios encountered in practice. For binary outcomes, the within-study variance decreases with increasing sample size, so large values of $\sigma_{\mathrm{i}}^{2}$ (close to 0:6) represent small trials included in the meta-analysis, and small values of $\sigma_{i}{ }^{2}$ represent large trials.

The effect size $y_{i}$ for $i=1, \ldots, k$ is generated from a normal distribution with mean $\mu$ and variance: $\sigma_{i}^{2}+\tau^{2}$

For each simulation of the meta-analysis, the confidence intervals based on the t-distribution and the [4] method are calculated, along with those of our proposed estimators "Modified Sidik-Jonkman Estimator (MSJE) [9]" are calculated along with those for "(Ord inary) Sidik-Jonkman Estimator (OSJE) [9]". The numbers of intervals containing the true $\mu$ are recorded for all four methods. The proportion of intervals containing the true $\mu$ (out of the 11000 runs) serves as the simulation estimate of the true coverage probability.

The results of the simulation study are presented in the tables (Nine Tables) in APPENDIX. From the tables, it can be seen that the coverage probabilities of the interval based on the t-distribution are larger than the coverage probabilities of the interval using the (Ordinary) DerSimonian and Laird method Estimator (ODLE) [4] for each $\tau^{2}$ and all values of $k$. Interestingly, our proposed estimator "Modified DerSimonian-Laird Estimator (MDLE) [4] performs even better than that. Although the coverage probabilities of the confidence interval from the $\mathrm{t}$-distribution, like other methods, are below the nominal level of 95 per cent, they are higher than the commonly applied interval based on the [4] method, particularly when $\mathrm{k}$ is small. This suggests that the simple confidence interval based on the $t$ distribution is an improvement compared to the existing simple confidence interval based on [4] method. Incidentally, 'MDLE' is the best. The most remarkable fact is that our proposed estimator "Modified SidikJonkman Estimator (MSJE) [9]" turns out to be the best in terms of the "Coverage Probability"!

\section{Conclusion}

As time is an important variable, and as Geospatial data would be temporal also, it is very significant to note that inasmuch as the 'time' could be viewed very conveniently in the shoes of 'space' the analogous treatment to 'Geotemporal' data in the meta-data set-up could well be carried out successfully, too! In fact the 'Geo-Spatial-Temporal' data in the meta-data setup dealt in two consecutive phases would be most realistic approach down-to-earth, as we have to carry out the analysis of 'Geospatial' data with this temporal toningup to be realistic. This is the direction of our proposed future work in the area, and could well be the source of enthusiasm for other researchers, particularly in the meta-data set-up.

\section{References}

[1] Armitage P. Controversies and achievements in clinical trials. Controlled Clinical Trials 1984; 5: 67-72.

[2] DerSimonian R, Laird N. Evaluating the effect of coaching on SAT Scores: a meta-analysis. Havard Ed Rev 1983; 53: 1-15.

[3] Brockwell, S E, and Gordon, I. R. A comparison of statistical methods for meta-analysis. Statistics in Medicine 2001; 20: 825-840.

[4] DerSimonian R, Laird N. Meta-analysis in clinical trials. Controlled Clinical Trials 1986; 7: 177-188.

[5] Halvorsen, $\mathrm{K}$ : Co mbining results fro $\mathrm{m}$ independent investigations: meta-analysis in medical research. In: Medical Uses of Statistics, Bailar, J C, Mosteteller, F; Eds. Boston: New Eng land Journal of Medicine (in press).

[6] Hardy, R J, Thompson, S. G. A likelihood approach to meta-analysis with random effects. Statistics in Medicine 1996; 15: 619-629.

[7] Olkin, I. Invited Commentary: Re: A critical look at some popular meta-analytic methods. American Journal of Epidemiology 1994; 140: 297-299.

[8] Sahai, Ashok \& Bhoendradatt Tewarie. MetaAnalysis of Spatial Panel Data for Socioeconomic Development Project(s) in India. Map World Forum (Jan. 2007 @ Hyderabad, ICC; INDIA).

[9] Sid ik, K, and Jonkman, J. N. A simple confidence interval for meta-analysis. Statistics in Medicine 2002; 21: 3153-3159.

[10] Villar, J, Maria E. Mackey, Guillermo, C. and Allan, D. Meta-analyses in systematic reviews of randomized controlled trials in perinatal medicine: comparison of fixed and random effect models. Statistics in Medicine 2001; 20: 3635-3647.

\section{Randomized Studies Panel Data CIs APPENDIX-I}

Number of Studies Seminal To Panel/Meta-Data Analysis:

$\underline{K}=10$. 
TABLE \# I.1::Performance Parameters Of CIs For $\tau^{2}=0.05 \&$ $1-\alpha=0.95$.

\begin{tabular}{|c|ccc|}
\hline CI Estimators $\downarrow$ & $\begin{array}{c}\text { Cvg.Prob. } \\
\text { LeftBias }\end{array}$ & $\begin{array}{l}\text { Cvg.Err. } \\
\text { RightBias }\end{array}$ & $\begin{array}{c}\text { Length } \\
\text { Rel.Bias }\end{array}$ \\
\hline ODLE & 0.905091 & 0.044909 & 0.456974 \\
Estimator & 5.000000 & 4.490909 & 0.053640 \\
\hline MDLE & 0.943364 & 0.006636 & 0.589395 \\
Estimator & 2.881818 & 2.781818 & 0.017657 \\
\hline OSJE & 0.927455 & 0.022545 & 0.519450 \\
Estimator & 3.790909 & 3.463636 & 0.045113 \\
\hline MSJE & 0.950364 & 0.000364 & 0.673215 \\
Estimator & 2.554545 & 2.409091 & 0.029304 \\
\hline
\end{tabular}

TABLE \# I.2::Performance Parameters Of CIs For $\tau^{2}=0.08 \&$ $\underline{1-\alpha=0.95}$.

\begin{tabular}{|c|ccc|}
\hline CI Estimators $\downarrow$ & $\begin{array}{c}\text { Cvg.Prob. } \\
\text { LeftBias }\end{array}$ & $\begin{array}{c}\text { Cvg.Err. } \\
\text { RightBias }\end{array}$ & $\begin{array}{c}\text { Length } \\
\text { Rel.Bias }\end{array}$ \\
\hline ODLE & 0.899636 & 0.050364 & 0.513184 \\
Estimator & 5.100000 & 4.936364 & 0.016304 \\
\hline MDLE & 0.943091 & 0.006909 & 0.673155 \\
Estimator & 2.981818 & 2.709091 & 0.047923 \\
\hline OSJE & 0.927455 & 0.022545 & 0.519450 \\
Estimator & 3.790909 & 3.463636 & 0.045113 \\
\hline MSJE & 0.950364 & 0.000364 & 0.673215 \\
Estimator & 2.554545 & 2.409091 & 0.029304 \\
\hline
\end{tabular}

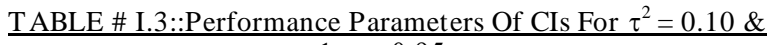
$\underline{1-\alpha=0.95}$.

\begin{tabular}{|c|ccc|}
\hline CI Estimators $\downarrow$ & $\begin{array}{c}\text { Cvg.Prob. } \\
\text { LeftBias }\end{array}$ & $\begin{array}{c}\text { Cvg.Err. } \\
\text { RightBias }\end{array}$ & $\begin{array}{c}\text { Length } \\
\text { Rel.Bias }\end{array}$ \\
\hline ODLE & 0.922455 & 0.027545 & 0.585853 \\
Estimator & 3.909091 & 3.845455 & 0.008206 \\
\hline MDLE & 0.943091 & 0.006909 & 0.673155 \\
Estimator & 2.981818 & 2.709091 & 0.047923 \\
\hline OSJE & 0.927455 & 0.022545 & 0.519450 \\
Estimator & 3.790909 & 3.463636 & 0.045113 \\
\hline MSJE & 0.950364 & 0.000364 & 0.673215 \\
Estimator & 2.554545 & 2.409091 & 0.029304 \\
\hline
\end{tabular}

Number of Studies Seminal To Panel/Meta-Data Analysis:

$\underline{\mathrm{K}=20 .}$

$\underline{\text { TABLE \# I.4.:Performance Parameters Of CIs For } \tau^{2}=0.05 \&}$ $1-\alpha=0.95$.

\begin{tabular}{|c|ccc|}
\hline CI Estimators $\downarrow$ & $\begin{array}{c}\text { Cvg.Prob. } \\
\text { LeftBias }\end{array}$ & $\begin{array}{c}\text { Cvg.Err. } \\
\text { RightBias }\end{array}$ & $\begin{array}{c}\text { Length } \\
\text { Rel.Bias }\end{array}$ \\
\hline \multirow{2}{*}{ ODLE Estimator } & 0.921273 & 0.028727 & 0.323959 \\
& 3.863636 & 4.009091 & 0.018476 \\
\hline \multirow{2}{*}{ MDLE Estimator } & 0.944000 & 0.006000 & 0.362095 \\
& 2.754545 & 2.845455 & 0.016234 \\
\hline \multirow{2}{*}{ OSJE Estimator } & 0.933273 & 0.016727 & 0.345697 \\
& 3.309091 & 3.363636 & 0.008174 \\
\hline \multirow{2}{*}{ MSJE Estimator } & 0.952727 & 0.002727 & 0.386560 \\
& 2.263636 & 2.463636 & 0.042308 \\
\hline
\end{tabular}


$\underline{1-\alpha=0.95}$.

\begin{tabular}{|c|ccc|}
\hline CI Estimators $\downarrow$ & $\begin{array}{c}\text { Cvg.Prob. } \\
\text { LeftBias }\end{array}$ & $\begin{array}{c}\text { Cvg.Err. } \\
\text { RightBias }\end{array}$ & $\begin{array}{c}\text { Length } \\
\text { Rel.Bias }\end{array}$ \\
\hline \multirow{2}{*}{ ODLE Estimator } & 0.919545 & 0.030455 & 0.364332 \\
& 4.054545 & 3.990909 & 0.007910 \\
\hline \multirow{2}{*}{ MDLE Estimator } & 0.945000 & 0.005000 & 0.409040 \\
& 2.754545 & 2.745455 & 0.001653 \\
\hline \multirow{2}{*}{ OSJE Estimator } & 0.933182 & 0.016818 & 0.388248 \\
& 3.372727 & 3.309091 & 0.009524 \\
\hline \multirow{2}{*}{ MSJE Estimator } & 0.956545 & 0.006545 & 0.435957 \\
& 2.163636 & 2.181818 & 0.004184 \\
\hline
\end{tabular}

TABLE \# I.6::Performance Parameters Of CIs For $\tau^{2}=0.10 \&$ $\underline{1-\alpha=0.95}$.

\begin{tabular}{|c|ccc|}
\hline CI Estimators $\downarrow$ & $\begin{array}{c}\text { Cvg.Prob. } \\
\text { LeftBias }\end{array}$ & $\begin{array}{c}\text { Cvg.Err. } \\
\text { RightBias }\end{array}$ & $\begin{array}{c}\text { Length } \\
\text { Rel.Bias }\end{array}$ \\
\hline \multirow{2}{*}{ ODLE Estimator } & 0.924455 & 0.025545 & 0.389048 \\
& 3.772727 & 3.781818 & 0.001203 \\
\hline \multirow{2}{*}{ MDLE Estimator } & 0.950909 & 0.000909 & 0.437252 \\
& 2.481818 & 2.427273 & 0.011111 \\
\hline \multirow{2}{*}{ OSJE Estimator } & 0.939909 & 0.010091 & 0.414913 \\
& 2.927273 & 3.081818 & 0.025719 \\
\hline \multirow{2}{*}{ MSJE Estimator } & 0.960727 & 0.010727 & 0.466361 \\
& 2.000000 & 1.927273 & 0.018519 \\
\hline
\end{tabular}

Number of Studies Seminal To Panel/Meta-Data Analysis:

$\underline{\mathrm{K}=60 .}$.

TABLE \# I.7::Performance Parameters Of CIs For $\tau^{2}=0.05 \&$ $\underline{1-\alpha=0.95}$.

\begin{tabular}{|c|ccc|}
\hline CI Estimators $\downarrow$ & $\begin{array}{c}\text { Cvg.Prob. } \\
\text { LeftBias }\end{array}$ & $\begin{array}{c}\text { Cvg.Err. } \\
\text { RightBias }\end{array}$ & $\begin{array}{c}\text { Length } \\
\text { Rel.Bias }\end{array}$ \\
\hline \multirow{2}{*}{ ODLE Estimator } & 0.939727 & 0.010273 & 0.188844 \\
& 3.145455 & 2.881818 & 0.043741 \\
\hline \multirow{2}{*}{ MDLE Estimator } & 0.946909 & 0.003091 & 0.195568 \\
& 2.727273 & 2.581818 & 0.027397 \\
\hline \multirow{2}{*}{ OSJE Estimator } & 0.942000 & 0.008000 & 0.192969 \\
& 3.045455 & 2.754545 & 0.050157 \\
\hline \multirow{2}{*}{ MSJE Estimator } & 0.949636 & 0.000364 & 0.199840 \\
& 2.627273 & 2.409091 & 0.043321 \\
\hline
\end{tabular}

TABLE \# I.8::Performance Parameters Of CIs For $\tau^{2}=0.08 \&$ $1-\alpha=0.95$.

\begin{tabular}{|c|ccc|}
\hline CI Estimators $\downarrow$ & $\begin{array}{c}\text { Cvg.Prob. } \\
\text { LeftBias }\end{array}$ & $\begin{array}{c}\text { Cvg.Err. } \\
\text { RightBias }\end{array}$ & $\begin{array}{c}\text { Length } \\
\text { Rel.Bias }\end{array}$ \\
\hline \multirow{2}{*}{ ODLE Estimator } & 0.943636 & 0.006364 & 0.214324 \\
& 2.772727 & 2.863636 & 0.016129 \\
\hline \multirow{2}{*}{ MDLE Estimator } & 0.953000 & 0.003000 & 0.221980 \\
& 2.290909 & 2.409091 & 0.025145 \\
\hline \multirow{2}{*}{ OSJE Estimator } & 0.948727 & 0.0012730 & 0.218528 \\
& 2.481818 & 2.645455 & 0.031915 \\
\hline \multirow{2}{*}{ MSJE Estimator } & 0.955636 & 0.005636 & 0.226334 \\
& 2.109091 & 2.327273 & 0.049180 \\
\hline
\end{tabular}
$1-\alpha=0.95$. 


\begin{tabular}{|c|ccc|}
\hline CI Estimators $\downarrow$ & $\begin{array}{c}\text { Cvg.Prob. } \\
\text { LeftBias }\end{array}$ & $\begin{array}{c}\text { Cvg.Err. } \\
\text { RightBias }\end{array}$ & $\begin{array}{c}\text { Length } \\
\text { Rel.Bias }\end{array}$ \\
\cline { 1 - 4 } & 0.941364 & 0.008636 & 0.228954 \\
\multirow{2}{*}{ ODLE Estimator } & 2.963636 & 2.900000 & 0.010853 \\
& 0.948000 & 0.002000 & 0.237134 \\
\multirow{2}{*}{ MDLE Estimator } & 2.572727 & 2.627273 & 0.010490 \\
\hline \multirow{2}{*}{ OSJE Estimator } & 0.944727 & 0.005273 & 0.233378 \\
& 2.854545 & 2.672727 & 0.032895 \\
\hline \multirow{2}{*}{ MSJE Estimator } & 0.951818 & 0.001818 & 0.241716 \\
& 2.527273 & 2.290909 & 0.049057 \\
\hline
\end{tabular}

\section{Bootstrapping}

\section{APPENDIX-II}

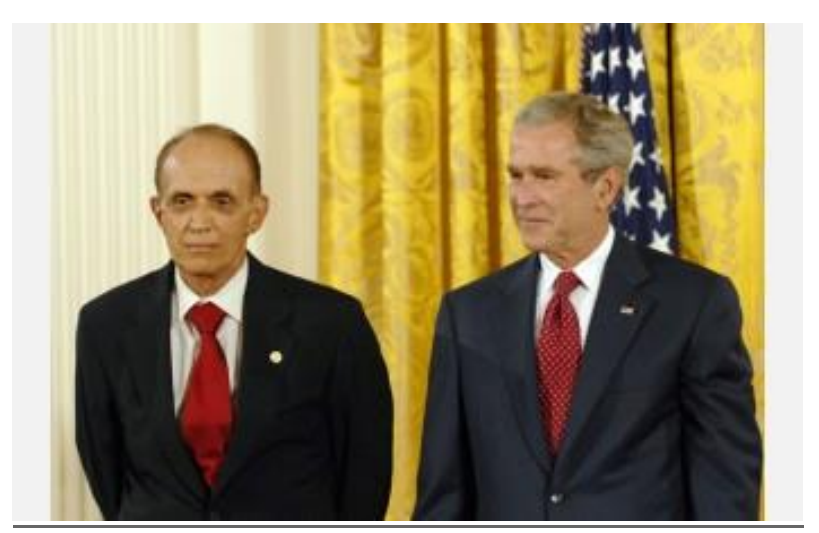

Bradley Efron (born May 24, 1938)[1] is an American statistician best known for proposing the bootstrap resampling technique, which has had a major impact in the field of statistics and virtually every area of statistical application. The bootstrap was one of the first computer-intensive statistical techniques, replacing traditional algebraic derivations with data-based computer simulations. He is currently a Professor of Statistics at Stanford University. At Stanford he has been the Chair of the Department of Statistics, Associate Dean of Science, Chairman of the University Advisory Board, Chair of the Faculty Senate and Chair of the Undergraduate Program in Applied Mathematics. Efron holds the Max H. Stein endowed chair as Professor of Humanities and Sciences at Stanford University.

Historica1/Mythological Use of The Word "Bootstrap" $\rightarrow \downarrow \downarrow \downarrow$

\section{Gromyko's Breakfast}



SATURDAY, MAY 8, 2010

Pulling Yourself Up By Your Bootstraps

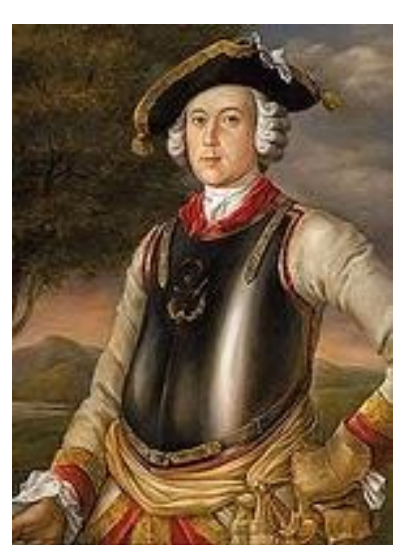

I guess I've always considered myself a "by the bootstraps" type of person. As it turns out, though, I may as well consider myself a cannonball-riding type of person or an Alexander the Great-defeating type of person.

"By the bootstraps" is such a ubiquitous phrase in modern American culture that one never pauses to consider what it actually means. "Pull yourself up by your bootstraps"? Really? It seems physically impossible. Which is exactly why the orig in of the phrase is linked to the German folk hero Baron Münchhausen--he who used cannonballs as a method of transportation and bested Alexander the Great, in addition to visiting the moon (once on purpose and once by accident). Before "by the bootstraps" was synonymous with good old fashioned American gumption, it was a phrase used to define something as absurdly impossible.

Much like Münchhausen's greatest exploits, the history of "by the bootstraps" is disputed. It has been commonly held that the phrase originated in a scene from The Surprising Adventures of Baron Munchausen in which the industrious baron pulls himself out of a swamp by using his bootstraps. The only problem is that the earliest German versions of the book have the baron using his ponytail, not his bootstraps, to escape the swamp. Many scholars now believe that the phrase is, fittingly, an American creation and some link its origin to a folk hero who's sort of the American Münchhausen--Davy Crocket. Instead of a swamp, it's held that Crocket pulled himself over a fence by his bootstraps and it's likely that "by the bootstraps" became part of the Münchhausen story through repeated English translations.

Whatever the case, this myth-build ing flourish came to be used throughout the 19th century as an example of a ridiculously impossible task, such as it appeared in an 1862 Chicago Tribune article: "The hopeful individual who expects to raise a weight vastly beyond his strength, belongs to the same class of fools with great expectations, as he who promises to lift himself by his boot straps." As Ben Zimmer noted in a recent discussion of the American Dialect Society, "The shift in the metaphor's sense to suggest a *possible* task 
doesn't seem to have occurred until the early 20th century."

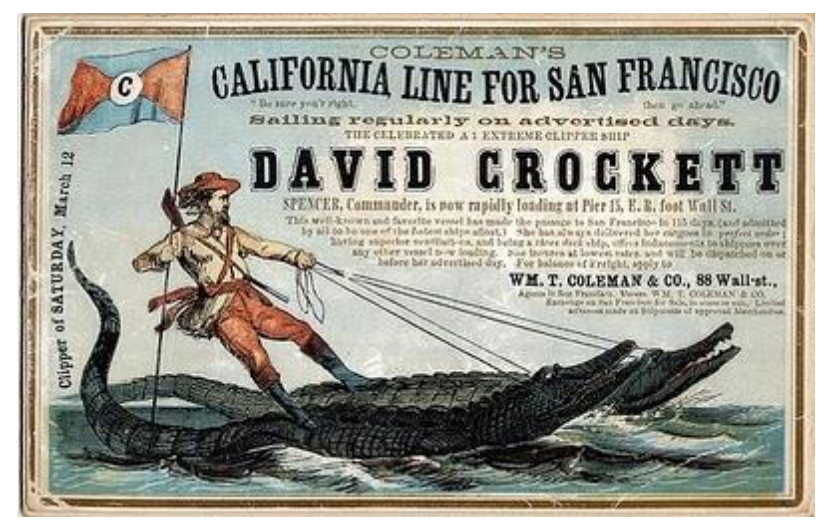

In 1922, the Oxford English Dictionary defined the figurative sense of "bootstrap" as "to raise or better oneself by one's own unaided efforts" and quoted a passage from James Joyce's Ulysses.

Joyce writes about those "who had forced their way to the top from the lowest rung by the aid of their bootstraps." Here, Joyce presents "bootstraps" in the new, 20th century sense of the phrase but he's also playing with its multiple layers of meaning (I mean, it is Ulysses). In this passage, Joyce uses two common metaphors for social advancement--the ladder and the bootstrap--and in tandem they work to show one's advancement both by and because of the use of bootstraps. Oftentimes one advances socially not by simply overcoming an obstacle through individual will but by exp loiting this original achieve ment to accelerate his/her continued advancement. In this way, "bootstrap" retains a connection to its mythic roots.

"American mythology fits all its greats with bootstraps," Alexander Ewing wrote in a recent essay for Intelligent Life. "Abraham Lincoln came from a backwoods farm, and some like to imagine Jefferson and Washington tilling the Virginia soil. Bootstrappers built steel mills in Pennsylvania, cars in Detroit and computers in Silicon Valley." Nowhere is the bootstrap mythology more prominent than in American politics, where candidates regularly trade on their humble orig in stories to climb the ranks of government. Covering the 2004 Democratic National Convention, "The Daily Show" poked fun at the bootstrap story one-upmanship of the speakers. Then-junior Illinois Senator Barack Obama was the clear winner as he described how his father "grew up herding goats." For some insight on the political posturing, Jon Stewart turned to Stephen Colbert, then the Chief Political Analyst for "The Daily Show." Colbert declared, "I believe in the promise of America that I, the son of a turf miner, the grands on of a goat ball-licker, could one day leave those worthless hicks behind while still using their story to enhance my own credibility."

Looking for a job after college, I added a few lines to the end of my resume to illustrate my bootstrap mentality. It included this boast: "I have been constantly employed at a long list of diverse jobs since I was 15 . I have laid asphalt, sold orthopedic shoes, held almost every restaurant position, worked as mover, a receptionist, a janitor, a tutor, and even an assistant to a Zamboni operator." In an interview at a production company, the producer chuckled about the Zamboni job. He looked down at my resume and said, "Yeah, that's a good line. You should keep that." Here's the truth: during high school, I once had a job at a local rec center On four, maybe five occasions, I helped the Zamboni driver shovel snow off the edge of the hockey rink. I got the job at the production company, though.

Notions \&amp; potions said... You tell a colorful tale, Andy!

This is fascinating! I think A merican society still has a strong sense of nostalgia and a desire to be connected to "the American dream," especially as the disconnect between the older generation of labor, the "working class," and today's young upstarts grows deeper...this may interest you?

http://en.wikipedia.org/wiki/The_Social_Contract/MAY 17, 2010 7:22 AM

Andrei Andreye vich Gromyko (1909 - 1989) was a Soviet politician and diplomat.

Gromyko's dour demeanor echoed throughout his tenure as Soviet foreign minister. There is a story that has Gromyko exiting a Washington hotel one morning and being asked by a reporter, "Minister Gromyko, did you enjoy your breakfast today?" His response was "Perhaps."

When I was growing up, my grandpa, a Midwestern businessman and sometimes disc jockey, always called me Gromyko.

My name is Andrew Gauthier/I live in New York City/ andrewjgauthier@gmail.com/twitter.com/and rewgauthier

$* * * * * * * * * * * * * * * * * * * * * * * * * * * * * * * * * * * * * * * * * * * * * * * * * * *$

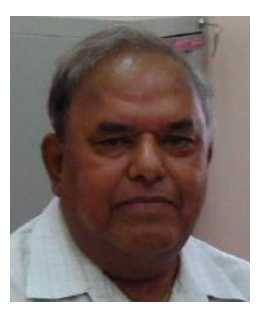

Ashok Sahai is working presently at The University of The West Indies; St. Augustine Campus (TRINIDAD \& TOBAGO) as a Professor of Statistics in the Department of Mathematics \& Statistics (Faculty of Science \& Agriculture) since February 2006.

Dr. Sahai started his teaching-n-research career as a Lecturer in Statistics Department at Lucknow University (INDIA) in July 1966, and continued thereat till April 1980. He has, up-to-date, published more than one hundred research papers in peer-reviewed journals of international repute \& in the peer-reviewed proceedings of international conferences.

He worked as Reader in Statistics and as Profess or of Statistics in the Department of Mathematics at 
University of Roorkee (Now IIT Roorkee) during the period: April 1980- July 1995. Prof. Sahai had also worked as an Assoc. Professor of Statistics at University of Dar-Es-Salaam; TANZANIA (East Africa) during the period: July 1982- June 1984, and as a Professor of Statistics at University of Swaziland (Southern Africa) during the period: July 1993 - June 2003. He worked as a Guest Scholar @ PharmacoEconomic Research Centre; University of Arizona, TUCSON during the period from July 2003 to October 2003 and as Visiting Professor @ Hyderabad; INDIA during December 2003 to January 2006 in ICFAI Tech. University, Medchel Rd.; in Aurora School of Management at Chikkadpally; and in St. Ann's P.G. (Management) College For Women at Mallapur.

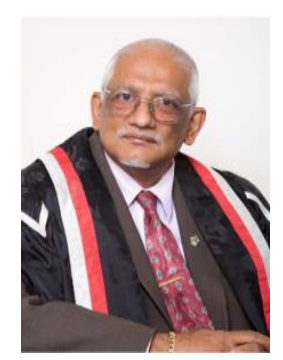

Clement K. Sankat is a member of the Executive Management Team of The University of the West Indies (UWI) and is currently Pro-ViceChancellor and Campus Principal of St. Augustine Campus since January $1,2008$. He is a great researcher and a great academic leader. He holds a Bachelor's Degree (First Class Honours) and Master's in Mechanical Engineering from the UWI and a Doctorate from the University of Guelph's School of Engineering (Canada). Prof. Sankat is Chartered Engineer (CEng), a Fellow of the Institution of Agricultural Engineers (FIAgrE) of the UK and a Fellow of the Association of Professional Engineers of Trinidad and Tobago (FAPETT). He has been Dean of the Faculty of Engineering \& Pro-ViceChancellor for Graduate Studies @ UWI.

He has been steadfast in supporting student centeredness and development, graduate-level training and in building capacity, promoting quality and accreditation for engineering education at the UWI in the Americas. Professor Sankat has for many years been actively involved in the application and promotion of Science and Technology ( $\mathrm{S} \& \mathrm{~T}$ ) for national and regional development. He is a long serving member on the Board of Directors of the Caribbean Industrial Research Institute (CARIRI). For his efforts in Public Service, University Service and Research he was awarded in 2001, the UWI Vice Chancellor's Award for Excellence. His accomplishments were also formally recognized by the University of New Brunswick which conferred on him an Honorary Doctoral Degree in 2010.

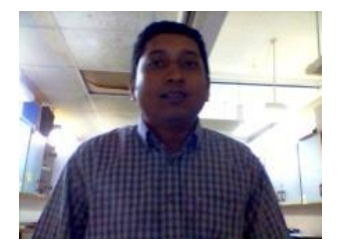

Koffka Khan was born in San Fernando, Trinidad and Tobago in 1978. He received the B.Sc. and M.Sc. degrees from University of the West Indies, in 2002 and 2008, respectively.
He was awarded by the University of the West Indies for his contributions made in postgraduate work in 2009 as a research assistant.

$\mathrm{He}$ is working presently at The University of The West Indies; St. Augustine Campus (TRINIDAD \& TOBAGO) as a Tutor in Computer Science in the Department of Computing and Information Technology (Faculty of Science \& Agriculture) since September 2006. Mr. Khan started his teaching-n-research career as a Demonstrator in Computer Science at the University of The West Indies at the Department of Mathematics and Computer Science. He has up-to-date, published ten research and co-authored four papers in journals of international repute $\&$ in the proceedings of international conferences.

How to cite this paper: Ashok Sahai,Clement K. Sankat,Koffka Khan,"Decision-Making Using Efficient Confidence-Intervals with Meta-Analysis of Spatial Panel Data for Socioeconomic Development Project-Managers", International Journal of Intelligent Systems and Applications(IJISA), vol.4, no.9, pp.92-103, 2012. DOI: 10.5815/ijisa.2012.09.12 